\title{
Alterations in Reward, Fear and Safety Cue Discrimination after Inactivation of the Rat Prelimbic and Infralimbic Cortices
}

\author{
Susan Sangha*,', Paul D Robinson', Quentin Greba', Don A Davies' and John G Howland*,' \\ 'Department of Physiology, University of Saskatchewan, Saskatoon, SK, Canada
}

\begin{abstract}
Accurate discrimination of environmental cues predicting reward, fear, or safety is important for survival. The prelimbic and infralimbic cortices are implicated in regulating reward-seeking and fear behaviors; however, no studies have examined their roles in discriminating among reward, fear, and safety cues. Using a discriminative conditioning task that includes presentations of a reward cue (paired with a reward pellet), fear cue (paired with footshock), and a compound fear + safety cue (no footshock) within the same sessions allowed us to assess the flexibility and precision of fear and reward-seeking behaviors to these cues. We found that fear behavior was appropriately limited to the fear cue in untreated rats, but during infralimbic cortical inactivation, similar levels of fear were seen to the fear and compound fear + safety cues. Reward-seeking behavior was also appropriately limited to the reward cue in untreated rats. Inactivating the prelimbic cortex altered discriminative reward seeking as rats with prelimbic inactivation did not increase their reward seeking behavior during the reward cue to the same degree as saline controls. Our results imply dissociable roles of the two cortical regions: the prelimbic cortex in precise discriminative reward seeking and the infralimbic cortex in discriminating between fear and safety cues. These data suggest that alterations in the balance of activity between areas homologous to the prelimbic and infralimbic cortices may be involved in the processes that go awry in anxiety and addiction disorders.

Neuropsychopharmacology (20I4) 39, 2405-24I3; doi: I 0.I038/npp.20 I4.89; published online I4 May 2014
\end{abstract}

\section{INTRODUCTION}

Learning to fear and avoid life-threatening stimuli is critical for survival but maladaptive when it persists in the absence of a direct threat. Accurately classifying stimuli as dangerous or safe is important in initiating the proper emotional response. Safety signals alert the organism to when an environment is safe thus promoting rewardseeking behaviors, such as feeding and mating, whereas fear signals inhibit these behaviors. Understanding how danger and safety cues are encoded in the brain is necessary to develop better treatments for those suffering from anxiety disorders (Kessler et al, 2005). Behavioral therapy is commonly used for treating anxiety disorders and often involves extinction, the repeated exposures to a fear cue in the absence of an aversive outcome. Safety conditioning is another method of reducing fear whereby a safety cue in conjunction with a fear cue signifies no aversive outcome, whereas the fear cue on its own results in an aversive outcome. Thus, fear extinction and safety conditioning are related but distinct (Christianson et al, 2012).

*Correspondence: Dr S Sangha or Dr JG Howland, Department of Physiology, University of Saskatchewan, GB33, Health Sciences Building, 107 Wiggins Road, Saskatoon, SK S7N 5E5, Canada, Tel: + I 306966 2032, Fax: + 306966 4298, E-mail: sangha@purdue.edu or john.howland@usask.ca

Received 28 November 2013; revised 4 April 2014; accepted 7 April 2014; accepted article preview online 14 April 2014
Posttraumatic stress disorder (PTSD) patients show impaired fear inhibition during the presentation of a combination of conditioned fear and safety cues (Jovanovic et al, 2009) as well as hypoactivity in the ventromedial prefrontal cortex (vmPFC) (Rauch et al, 2006). Thus, the vmPFC is hypothesized to be a site of pathophysiology in PTSD and may contribute to safety signaling. In rats, lesions (Gewirtz et al, 1997) or temporary inactivation (Christianson et al, 2008) of the vmPFC do not interfere with behavioral responses to safety signals. However, the rat vmPFC consists of two distinct sub-regions, the prelimbic (PL) and infralimbic (IL) cortices (Brown and Bowman, 2002; Heidbreder and Groenewegen, 2003; Kesner and Churchwell, 2011; Uylings et al, 2003; Vertes, 2006). Recent studies show that these sub-regions have opposing roles in fear behavior: PL mediates fear expression, whereas IL mediates fear reduction during extinction (Burgos-Robles et al, 2009; Corcoran and Quirk, 2007; Sierra-Mercado et al, 2011; Vidal-Gonzalez et al, 2006). These opposing roles may explain conflicting reports of vmPFC inactivations impairing (Laurent and Westbrook, 2009; Sierra-Mercado et al, 2006) and facilitating (Akirav et al, 2006) fear extinction. Given the work dissociating the roles of IL and PL in retrieving fear extinction memory, we hypothesize that a similar dissociation of PL and IL function may exist in regulating fear to safety cues.

Dissociable roles in reward-seeking behavior have also been reported for the PL and IL. For example, a double dissociation exists for the effects of PL and IL lesions on the 
sensitivity to devaluation on a discriminable instrumental response task whereby PL lesions impair sensitivity to goal values and IL lesions facilitate their sensitivity even after extended training (Killcross and Coutureau, 2003). In addition, IL inactivation impairs reward collection, whereas PL inactivation does not (Burgos-Robles et al, 2013). PL is required in guiding appropriate reward-seeking behavior for cocaine (Mihindou et al, 2013) and food rewards (Marquis et al, 2007) in tasks that rely on discrimination among environmental cues. The PL and IL have also been associated with reinstatement of drug seeking and the extinction of drug seeking, respectively (Van den Oever et al, 2010). However, how the PL and IL guide reward seeking when discriminating among conditioned reward, fear, and safety cues is unknown.

To test for a functional dissociation between the PL and IL in fear, safety, and reward discrimination, we temporarily inactivated these areas in rats by performing a recently developed discriminative Pavlovian conditioning task requiring rats to flexibly switch their fear and reward-seeking behavior in response to reward, fear, or safety cues (Sangha et al, 2013). In addition, we analyzed the effects of PL and IL inactivation during subsequent extinction to the fear and reward cues.

\section{METHODS}

\section{Subjects}

Thirty-six Long Evans male rats (Charles River Laboratories, Quebec, Canada) weighing 300-350g were single housed (12-h light/dark cycle, lights on at 0700 hours) and handled for 1 week. Rats had ad libitum access to food and water up until the third reward learning session, when they were restricted to 22-24 g of food per day for the remainder of the experiment. All experiments were in accordance with the Canadian Council on Animal Care and approved by the University of Saskatchewan Animal Care and Use Program.

\section{Surgery}

Rats were anesthetized with isoflurane and stereotaxically implanted bilaterally with stainless steel 23-gauge guide cannula either dorsal to the PL (AP +3.20 mm; ML + I$0.70 \mathrm{~mm}$; DV $-3.60 \mathrm{~mm}$ ) or IL (AP + 3.20 mm; ML + I $0.50 \mathrm{~mm}$; DV $-4.20 \mathrm{~mm}$ ) (Paxinos and Watson, 1997). During infusions, needles (30-gauge) extended $1 \mathrm{~mm}$ beyond the guide cannulas into either the PL or IL (Figure $1 \mathrm{~b}$ and $\mathrm{c}$ depicts the location of the infusion sites). Rats were allowed 7-10 days to recover. Stainless steel 28gauge dummy cannulas were inserted into the guide cannulae between infusions. Infusion sites were confirmed using conventional methods (Cazakoff and Howland, 2011). Fourteen rats were excluded due to one or both infusion sites being located outside the PL or IL or at the boundary between the PL and IL. After exclusion, 10 rats with bilateral PL cannulae and 12 rats with bilateral IL cannulae were included.

\section{Infusions}

Muscimol and baclofen (Sigma-Aldrich, Canada) were dissolved separately in PBS $(500 \mathrm{ng} / \mu \mathrm{l})$ and mixed together
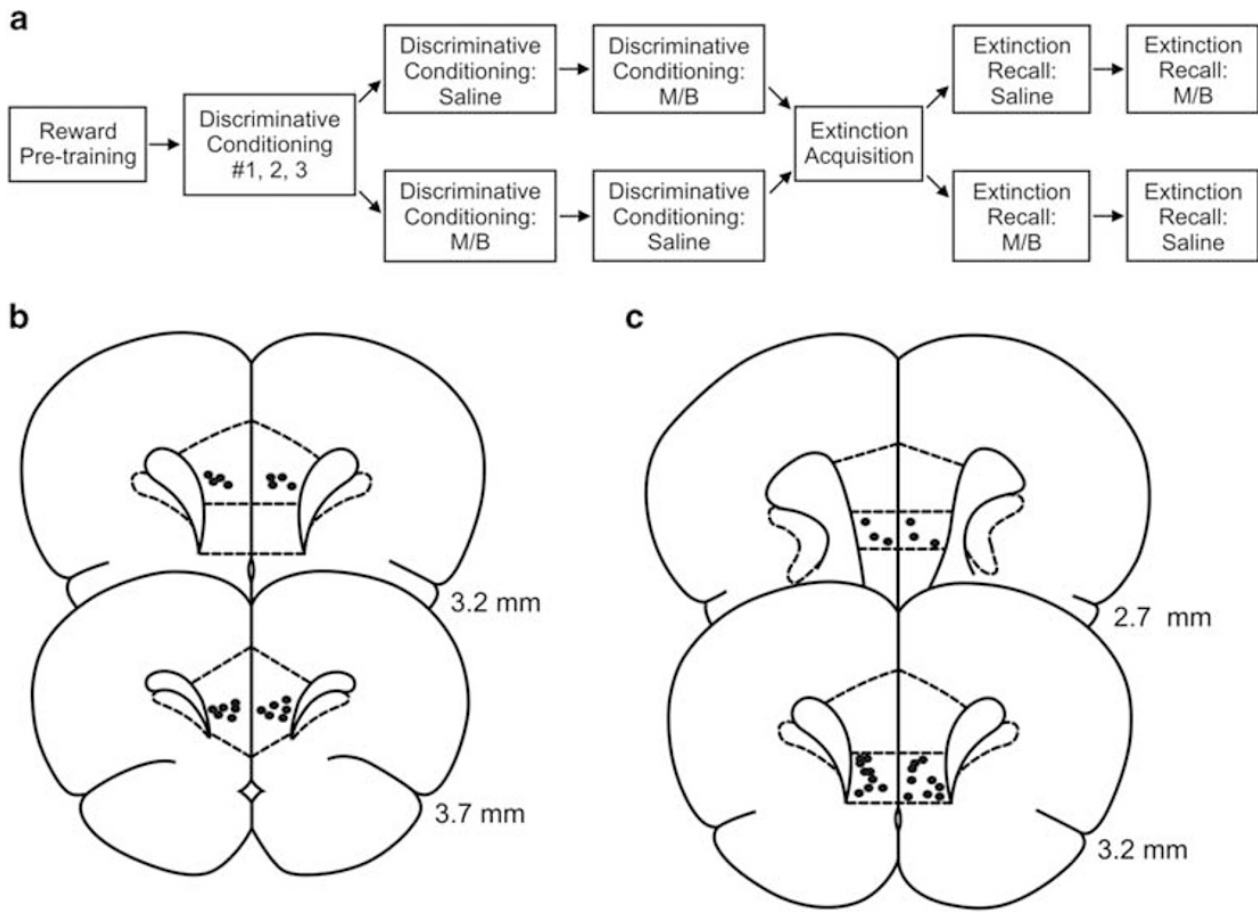

Figure I (a) Schematic of the experimental design. Rats were pretrained on the reward cue-reward pellet association followed by three discriminative conditioning sessions in which the reward cue ( + reward pellet), fear cue ( + footshock), compound fear + safety cues (no footshock), and safety cue (no footshock) were presented. Rats then received either saline or muscimol/baclofen (M/B) infusions during another discriminative conditioning session followed by the opposite drug treatment the next day. During extinction acquisition, all rats received unreinforced presentations of the fear and reward cues. Rats then received either saline or M/B infusions during an extinction recall test followed by the opposite drug treatment the next day. (b) Infusion needle placements in the prelimbic cortex (PL). (c) Infusion needle placements in the infralimbic cortex (IL). In panels b and c, numbers indicate the distance of the histology plate anterior to bregma. 
in a 1:1 ratio (McFarland and Kalivas, 2001; St Onge and Floresco, 2010). This muscimol/baclofen mixture (M/B) was administered 20 min before testing to enhance $\mathrm{GABA}_{\mathrm{A}}$ and $\mathrm{GABA}_{\mathrm{B}}$ receptor activity, thereby inactivating target structures. Sham infusions were performed on the first three discriminative conditioning sessions to habituate rats to the infusion procedure. Two drug treatment days followed a within-subjects design. Previous studies infusing $0.5 \mu \mathrm{l}$ of $\mathrm{M} / \mathrm{B}$ has been successfully used in dissociating the influence of PL and IL on behavior (Marquis et al, 2007; Willcocks and McNally, 2013), and thus in our study, rats were infused bilaterally with $0.5 \mu \mathrm{l}(60 \mathrm{~s})$ of $\mathrm{M} / \mathrm{B}$ or saline on the first drug treatment day. On the second drug treatment day, the drug treatments were reversed. After the infusion was completed, injectors were left in place for $1.5 \mathrm{~min}$ to allow for drug diffusion. A similar within-subjects design was implemented during the two tests of extinction recall (drug delivery order was maintained). No significant order effects were observed (unpaired $t$-tests, $p>0.05$ ) so the data were collapsed by treatment.

\section{Behavioral Apparatus}

Standard operant chambers (ENV-008; Med Associates, St Albans, VT) encased in sound-attenuating cubicles were used. Reward pellets were delivered into a recessed port $2.5 \mathrm{~cm}$ above the floor in the center of one wall. Port entries and exits were monitored via an infrared beam. Two lights $(28 \mathrm{~V}, 100 \mathrm{~mA})$ located $10 \mathrm{~cm}$ from the floor on the same wall as the port served as the 20 -s continuous light cue. A light $(28 \mathrm{~V}, 100 \mathrm{~mA})$ located $18 \mathrm{~cm}$ above the floor on the wall opposite the port provided constant illumination. Auditory cues were delivered via a high-frequency speaker (ENV-224BM) located $16 \mathrm{~cm}$ from the floor on the same wall as the port. Footshock $(0.5 \mathrm{~s}, 0.45 \mathrm{~mA})$ was delivered through a grid floor via a constant current aversive stimulator (ENV$414 S$ ). A video camera located on the door of the soundattenuating cubicle recorded the rat's behavior.

\section{Behavioral Training}

Rats were trained as described in Sangha et al (2013), with some modifications (Figure 1a). Rats were trained to associate an auditory cue $(11 \mathrm{kHz}, 200 \mathrm{~ms}$ on, $200 \mathrm{~ms}$ off for $20 \mathrm{~s} ; 70 \mathrm{~dB}$ ) with delivery of a reward pellet (Dust-less Precision Pellets, $45 \mathrm{mg}$, Rodent Purified Diet; BioServ, Frenchtown, NJ) (Reward Pretraining; pellet delivered pseudorandomly between 10 and $20 \mathrm{~s}$ after reward cue onset for 25 trials; intertrial interval (ITI), 90-130 s). The fifth reward conditioning session included five unreinforced presentations of each of the future fear and safety cues in order to habituate the rats to their presentation, thereby reducing any baseline freezing to these novel cues. Rats then had three discriminative conditioning sessions in which the reward cue-pellet association was maintained (pellet delivery at reward cue offset; 15 trials). Another auditory cue $(3 \mathrm{kHz}, 20 \mathrm{~s}, 70 \mathrm{~dB})$ was paired with a mild $0.45 \mathrm{~mA}, 0.5 \mathrm{~s}$ footshock and served as the fear cue (shock at cue offset, 4 trials). In separate trials, the 20 -s fear cue was simultaneously presented (ie, as a compound cue) with a 20-s safety cue (one $28 \mathrm{~V}, 100 \mathrm{~mA}$ light located on each side of the port) resulting in no footshock (15 trials). Trials in which the safety cue was presented alone without any footshock were also included (10 trials) to assess whether freezing developed to the safety cue as well as providing the animal with additional trials that contained a safety cue-no shock contingency. Trials were presented pseudorandomly (ITI, 100-140 s). During the tests for discriminative conditioning (Session \#4; Figures 2b, 3b, 4b, and 5b), infusions were performed to inactivate either the PL or IL before assessing memory for learned reward, fear, and safety cues. All reward and fear trials during the tests for discriminative conditioning were reinforced with food pellets and footshocks, respectively. One day later, rats underwent one session of extinction training (extinction acquisition) drugfree in which both the fear and reward cues were presented pseudorandomly without reinforcement. Beginning the next day, during the tests for extinction recall, infusions were repeated over 2 days. None of the trials during the tests for extinction recall were reinforced with either food pellets or footshocks. Note that a computer error resulted in the loss of fear and reward data during extinction recall (saline treatment) for three rats in the PL group.

\section{Behavioral Analyses and Statistics}

Cue-triggered effects on fear and reward behavior were assessed as follows (Morrow et al, 2011; Pecina et al, 2006). Fear behavior was assessed offline from videos by measuring freezing, defined as complete immobility with the exception of respiratory movements, which is an innate defensive behavior (Blanchard and Blanchard, 1969). The total number of seconds spent freezing was quantified during the entire $20 \mathrm{~s}$ of each cue presentation every $1 \mathrm{~s}$. This value was then normalized to the precue period: ((total number of seconds freezing during the 20-s cue-total number of seconds freezing during the 20-s immediately before cue presentation) $/ 20 \times 100$ ). $\mathrm{M} / \mathrm{B}$ infusions did not significantly alter freezing levels in the precue period (paired $t$-tests $p>0.05$ ) for rats with placements in either the PL (Saline $=1.9 \pm 1.4 \%, \quad M / B=0.0 \pm 0.0 \%) \quad$ or $\quad$ IL (Saline $=1.8 \pm 1.8 \%, \mathrm{M} / \mathrm{B}=0.6 \pm 0.4 \%$ ). Reward behavior was assessed by calculating the total time during which the rat's head was in the reward port during the entire $20 \mathrm{~s}$ of each cue presentation. This value was then normalized to the precue period: ((total time in port during the 20-s cue - total time in port during the 20 -s immediately before cue presentation) $/ 20 \times 100$ ). M/B infusions did not significantly alter the amount of time spent in the port in the precue period (paired $t$-tests $p>0.05$ ) for rats with placements in either the PL (Saline $=19.0 \pm 2.6 \%, M / B=$ $23.6 \pm 3.1 \%$ ) or IL (Saline $=13.3 \pm 2.3 \%, M / B=17.7 \pm 3.0 \%)$. Behavioral scoring was performed by four individuals blind to cannula placement and drug treatment; Pearson's correlations of freezing and reward behavior values obtained among the scorers were greater than $r=0.9$. Behavioral data were analyzed using one sample $t$-tests, paired $t$-tests, one- and two-way repeated-measures ANOVAs, and Tukey post-hoc tests where appropriate. One rat from the PL group and two rats from the IL group could not be used in the paired $t$-test analysis of the fold-preference ratios (Figures $3 b$ and $5 b$ ) as they did not enter the reward port during the non-reward cues. 

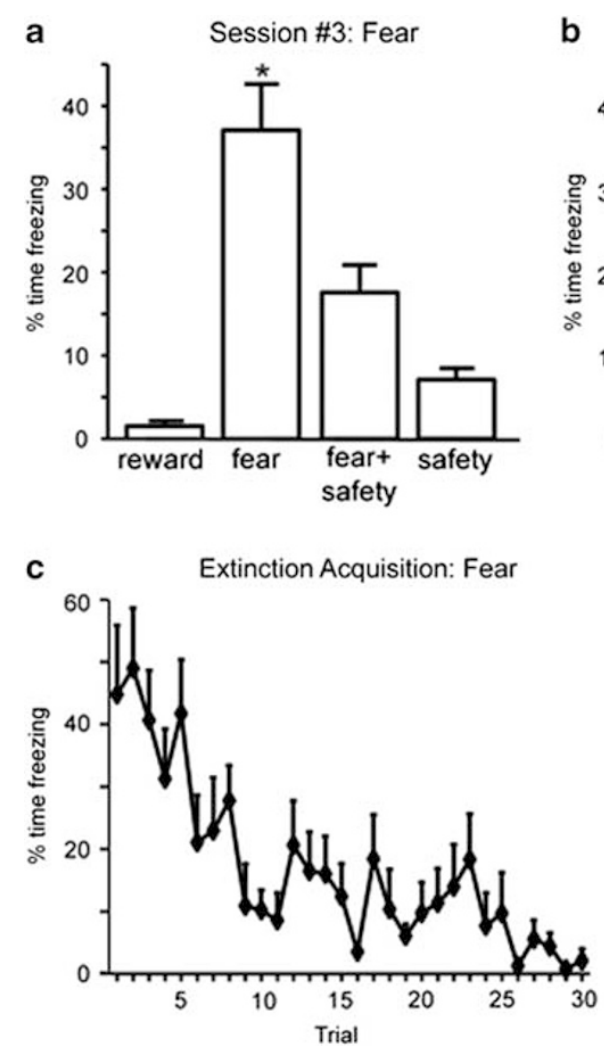
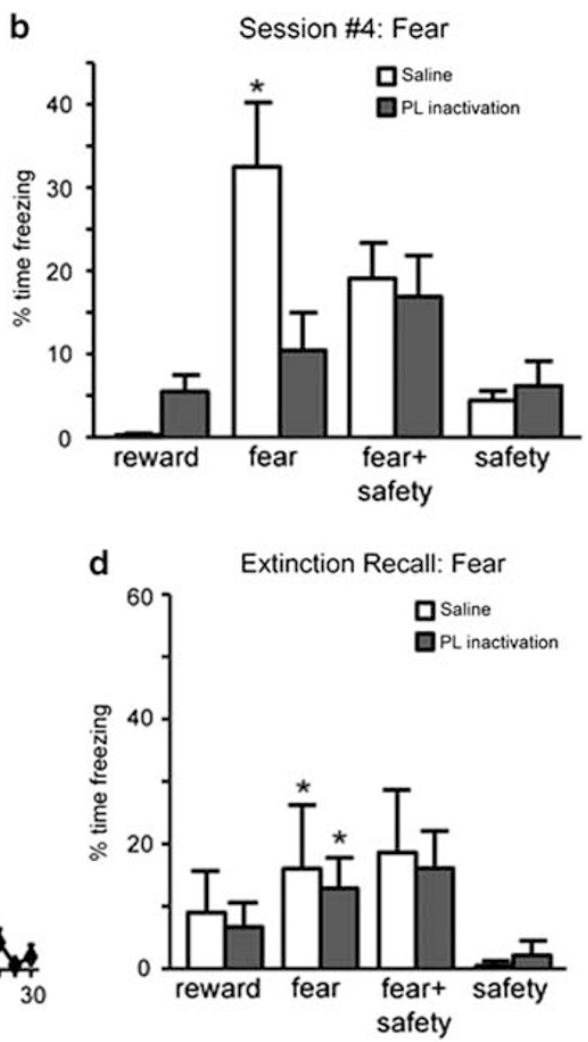

Figure 2 Prelimbic (PL) inactivation impairs fear expression. (a) Reduced freezing in the presence of the safety cue under drug-free conditions. Averaged percentage of time freezing was significantly higher during the fear cue compared with all other cues $(*<<0.05)$. (b) Saline-treated rats froze significantly more to the fear cue than to any other cue $(* p<0.05)$. PL inactivated rats froze significantly less to the fear cue when compared with saline-treated rats $(* p<0.05)$. (c) Significant within-session extinction of freezing behavior to the fear cue under drug-free conditions. (d) During extinction recall, both salinetreated and PL-inactivated rats showed a significant reduction in freezing to the fear cue compared with the beginning of extinction acquisition $(* p<0.05)$, demonstrating good fear extinction recall.

\section{RESULTS}

\section{PL Inactivation Impairs Fear Expression and Discriminative Reward Seeking}

Before infusions, rats with cannula implanted dorsal to the PL $(n=10)$ demonstrated significantly less freezing in the presence of the safety cue than the fear cue (Figure $2 a$ ). The percentage of time freezing was significantly higher during the fear cue compared with all other cues $(\mathrm{F}(3,27)=30.61, p<0.001$; post-hoc, $p<0.05)$. During the tests for discriminative conditioning following infusions (Figure 2b), there was no significant main effect of treatment $(\mathrm{F}(1,9)=3.88, p=0.81)$ but a significant main effect of cue $(\mathrm{F}(3,27)=8.51)$ and a treatment by cue interaction $(\mathrm{F}(3,27)=9.16, p<0.001)$. Following saline infusions, freezing was significantly higher during presentation of the fear cue than any other cue (post-hoc, $p<0.05$ ). PL inactivation significantly reduced freezing during the fear cue when compared with saline treatment (post-hoc, $p<0.05$ ). No significant differences in freezing were noted among any of the cues following PL inactivation (post-hoc, NS).

The same rats also demonstrated significant discriminative reward seeking during the reward cue before infusions (Figure 3a). Averaged reward seeking as measured by time spent in the port was significantly higher during the reward cue compared with all other cues before infusions
(Figure 3a; $\mathrm{F}(3,27)=33.87, p<0.001 ;$ post-hoc, $p<0.05)$. During the tests for discriminative conditioning following infusions (Figure $3 \mathrm{~b}$, left), there was a significant main effect of cue $(\mathrm{F}(3,27)=23.46, p<0.001)$ and treatment by cue interaction $(\mathrm{F}(3,27)=14.77, p<0.001)$ but no significant main effect of treatment $(\mathrm{F}(1,9)=1.98, p=0.19)$. Following saline infusions, time spent in the port was significantly higher during the reward cue compared with all other cues (post-hoc, $p<0.05$ ). During PL inactivation, the time spent in the port during the reward cue was also significantly higher than the other cues on that day, indicating discrimination among the cues. However, when the PL was inactivated, rats spent significantly less time in the reward port than following saline infusions ( $p o s t-h o c, p<0.05$ ) suggesting that discrimination is not as robust following PL inactivation. Next, we considered the fold preference or ratio of time spent in the port during the reward $v s$ non-reward cues (Figure $3 \mathrm{~b}$, right). When PL rats were treated with saline, they showed a 7.2-fold preference for the port during the reward cue compared with non-reward cues (ie, $\sim 46 \%$ time in port during reward cue $v \mathcal{S} \sim$ average $7 \%$ time in port during the non-reward cues). When the same PL rats were infused with $M / B$, they showed a 2.7 -fold increase in port time during the reward cue compared with the non-reward cues. Although the rats displayed a significant fold preference regardless of treatment (one sample $t$-test, saline 

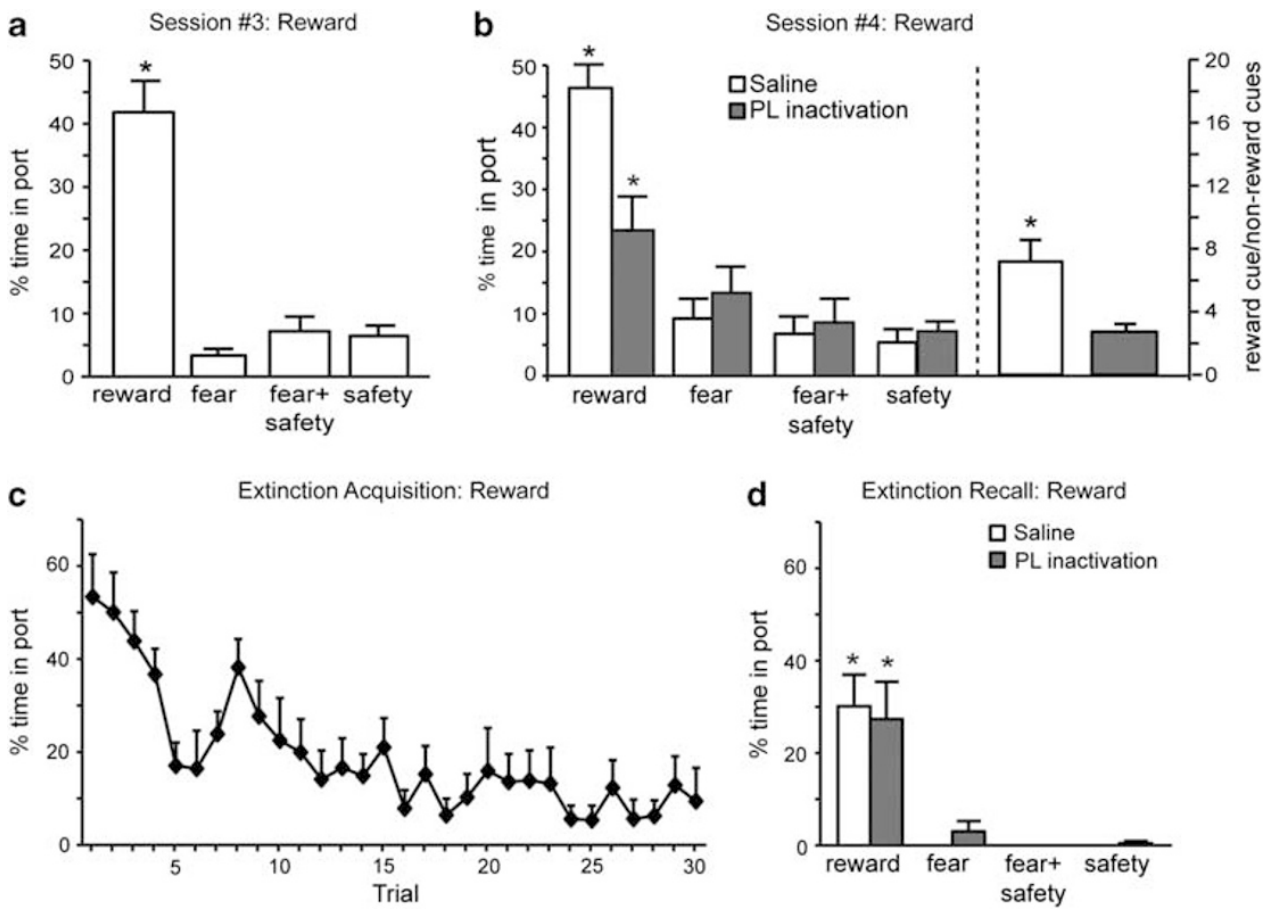

Figure 3 Prelimbic $(\mathrm{PL})$ inactivation impairs discriminative reward seeking. (a) Discriminative reward seeking under drug-free conditions. Percentage of time spent in port was significantly higher during the reward cue compared with all other cues $(* p<0.05)$ under drug-free conditions. (b) (left) Saline-treated rats spent significantly more time in the port during the reward cue compared with all other cues $(* p<0.05)$. PL inactivated rats also spent significantly more time in port during the reward cue compared with all other cues $(* p<0.05)$, but it was significantly less time than the saline-treated rats spent $(* 2<0.05)$. (b) (right) The ratio of time spent in port during the reward cue vs non-reward cues was calculated for saline-treated and PL-inactivated rats in order to assess the degree of discriminative reward seeking. The ratio for saline-treated rats was significantly higher than PL-inactivated rats (* $p<0.05)$, indicating that $\mathrm{PL}$ inactivation reduced the level of discrimination. (c) Significant within-session extinction of reward-seeking behavior to the reward cue under drug-free conditions. (d) During extinction recall, both saline-treated and PL-inactivated rats showed a significant reduction in reward seeking to the reward cue compared with the beginning of extinction acquisition $(* p<0.05)$, demonstrating good reward extinction recall.

$t(8)=4.50, p=0.002 ; \mathrm{M} / \mathrm{B} t(9)=3.56, p=0.006)$, the fold preference was significantly lower following $M / B$ treatment than saline treatment consistent with impaired discrimination $(t(8)=3.00, p=0.017)$.

One day later, the same rats underwent fear and reward extinction acquisition without treatment (Figure 1a). Significant within-session extinction of freezing and rewardseeking behavior during the fear and reward cues was observed (Figures $2 c$ and $3 c$ ). Percentage of time freezing and percentage of time spent in port during the last fear and reward cues, respectively, were significantly lower than the first fear and reward cue presentations (paired $t$-tests, $p<0.01$ ). During the test for extinction recall (Figure $2 \mathrm{~d}$ ), a significant reduction in freezing during the fear cue compared with the beginning of extinction acquisition (paired $t$-tests, $p<0.05$ ) was observed following either saline infusions or PL inactivation. No differences in freezing were detected between drug conditions for any cue (main effect treatment: $\mathrm{F}(1,6)=1.02$; main effect cue: $\mathrm{F}(3,18)=1.79$; treatment by cue interaction: $\mathrm{F}(3,18)=0.24$; all $p$ 's $>0.05)$. Both saline infusion and $\mathrm{PL}$ inactivation yielded a significant reduction in reward seeking to the reward cue compared with the beginning of extinction acquisition (paired $t$-tests, $p<0.05$ ). Similarly, no differences in reward seeking were detected between treatments for any cue during the tests for extinction recall (Figure $3 \mathrm{~d}$; main effect of treatment: $\mathrm{F}(3,18)=0.15$; treatment by cue interaction: $\mathrm{F}(3,18)=0.15$; both $\mathrm{p}$ 's $>0.05)$. Thus, PL inactivation did not significantly affect recall of either fear or reward extinction.

\section{IL Inactivation Impairs Fear and Safety Cue Discrimination and Recall of Fear Extinction}

Before infusions, rats implanted with cannula dorsal to the IL $(n=12)$ demonstrated significantly less freezing in the presence of the safety cue than the fear cue (Figure 4a). The percentage of time freezing was significantly higher during the fear cue compared with all other cues $(\mathrm{F}(3,33)=120.28, p<0.001$; post-hoc, $p<0.05)$. During the tests for discriminative conditioning (Figure $4 \mathrm{~b}$ ), a significant main effect of cue $(\mathrm{F}(3,33)=39.10, p<0.001)$ and cue by treatment interaction were observed $(\mathrm{F}(3,33)=3.26$, $p=0.034)$, with no main effect of treatment $(\mathrm{F}(1,11)=0.15$, $p=0.70)$. Following saline infusions, freezing was significantly higher during the fear cues than the other types of cues (post-hoc, $p<0.05$ ). This discrimination was not seen when the IL was inactivated: freezing levels to the fear cue and to the fear + safety cues were not significantly different (posthoc, N.S). Thus, IL inactivation impaired fear and safety cue discrimination. A comparison of freezing during the fear cue between treatments revealed that $\mathrm{M} / \mathrm{B}$ infusions into the IL 

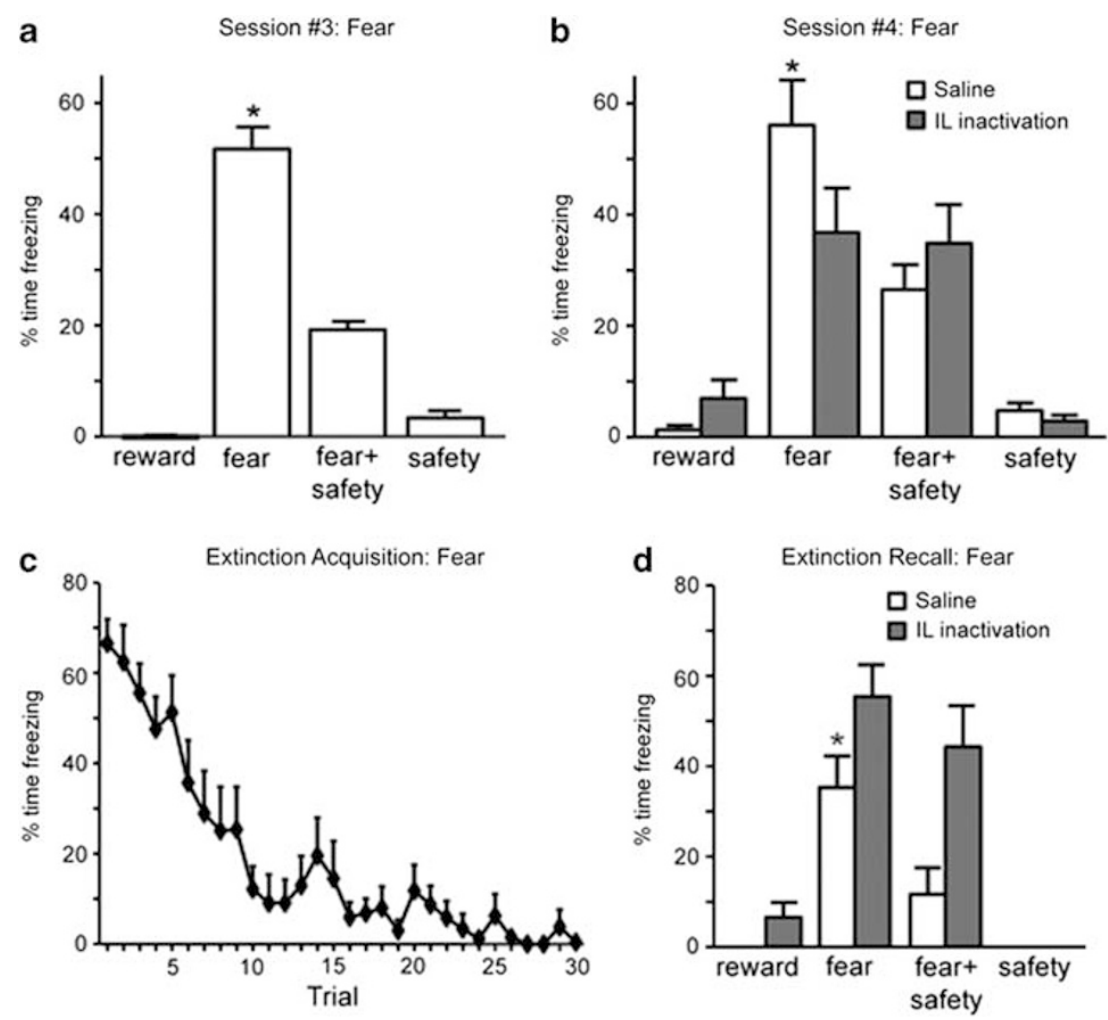

Figure 4 Infralimbic (IL) inactivation impairs fear and safety cue discrimination and recall of fear extinction. (a) Reduced freezing in the presence of the safety cue under drug-free conditions. Freezing was significantly higher during the fear cue compared with all other cues $(* p<0.05)$. (b) During session 4 , saline-treated rats froze significantly more to the fear cue than to any other cue $(* p<0.05)$. Freezing was not significantly different during presentations of either the fear cue or compound fear + safety cue during IL inactivation. (c) Significant within-session extinction of freezing behavior to the fear cue under drug-free conditions. (d) During extinction recall, saline-treated rats showed a significant reduction in freezing to the fear cue compared with the beginning of extinction acquisition $(* p<0.05)$. IL inactivation resulted in freezing levels to the fear cue similar to the beginning of extinction acquisition, demonstrating poor fear extinction recall.

significantly reduced freezing compared with levels observed following saline infusions (post-hoc, $p<0.05$ ).

The same rats also demonstrated significant discriminative reward seeking during the reward cue before infusions (Figure 5a). Averaged reward seeking as measured by the time spent in the port was significantly higher during the reward compared with all other cues before infusions (Figure 5a; $\mathrm{F}(3,33)=62.74, p<0.001$; post-hoc, $p<0.05$ ). During the tests for discriminative conditioning after the infusions were conducted (Figure 5b, left), significant main effects of treatment $(\mathrm{F}(1,11)=14.72, p<0.003)$, cue $(\mathrm{F}(3,33)=35.57, \quad p<0.001)$, and a significant cue by treatment interaction $(\mathrm{F}(3,33)=6.50, p<0.001)$ were found. Following either saline or $\mathrm{M} / \mathrm{B}$ infusions, time spent in the port was significantly higher during the reward cue compared with all other cues (post-hoc, $p<0.05$ ), indicating discrimination among the cues. When a ratio of time in port during the reward and non-reward cues was considered, IL rats treated with saline (10.2-fold preference, one sample $t$-test, $t(10)=$ $4.20, p=0.002)$ or $\mathrm{M} / \mathrm{B}$ (13.5-fold preference, one sample $t$-test, $t(10)=2.31, p=0.044)$ displayed similar patterns of increased time in port during the reward cue (saline $v s \mathrm{M} / \mathrm{B}$ treatment, $t(9)=-0.72, p=0.49$ ). Thus, although IL inactivation dampened the percentage of time in the reward port for all cues compared with controls, a similar fold preference for the reward port existed. during IL inactivation.
One day later, the rats underwent fear and reward extinction (Figure 1a). Significant within-session extinction of freezing and reward-seeking behavior during the fear and reward cues was observed (Figures $4 \mathrm{c}$ and $5 \mathrm{c}$ ). Percentage of time freezing and percentage of time spent in port during the last fear and reward cues, respectively, were significantly lower than the first fear and reward cue presentations (paired $t$-tests, $p<0.01$ ). During extinction recall, differences in freezing were detected between drug treatments (Figure $4 \mathrm{~d}$ ). Following saline infusions, rats showed a significant reduction in freezing to the fear cue compared with the beginning of extinction acquisition (paired $t$-test, $p<0.05$ ), demonstrating good fear extinction recall. In contrast, IL inactivation resulted in freezing levels to the fear cue similar to the beginning of extinction acquisition (paired $t$-test, $p>0.05$; Figure $4 \mathrm{~d}$ ). Thus, IL inactivation impaired recall of fear extinction. During extinction recall, significant main effects of cue $(\mathrm{F}(3,33)=42.54, p<0.001)$, treatment $(\mathrm{F}(1,11)=9.17, p=0.011)$, and a cue by treatment interaction $(\mathrm{F}(3,33)=4.55, p=0.009)$ were observed. Posthoc analyses indicated that when the rats were infused with $M / B$, they showed significantly higher freezing to the fear cue than following saline infusions $(p<0.05)$. In addition, freezing to the fear + safety cue was significantly higher following $\mathrm{M} / \mathrm{B}$ infusions than saline infusions (post-hoc, $p<0.05$ ). Finally, it is noteworthy that significantly less 

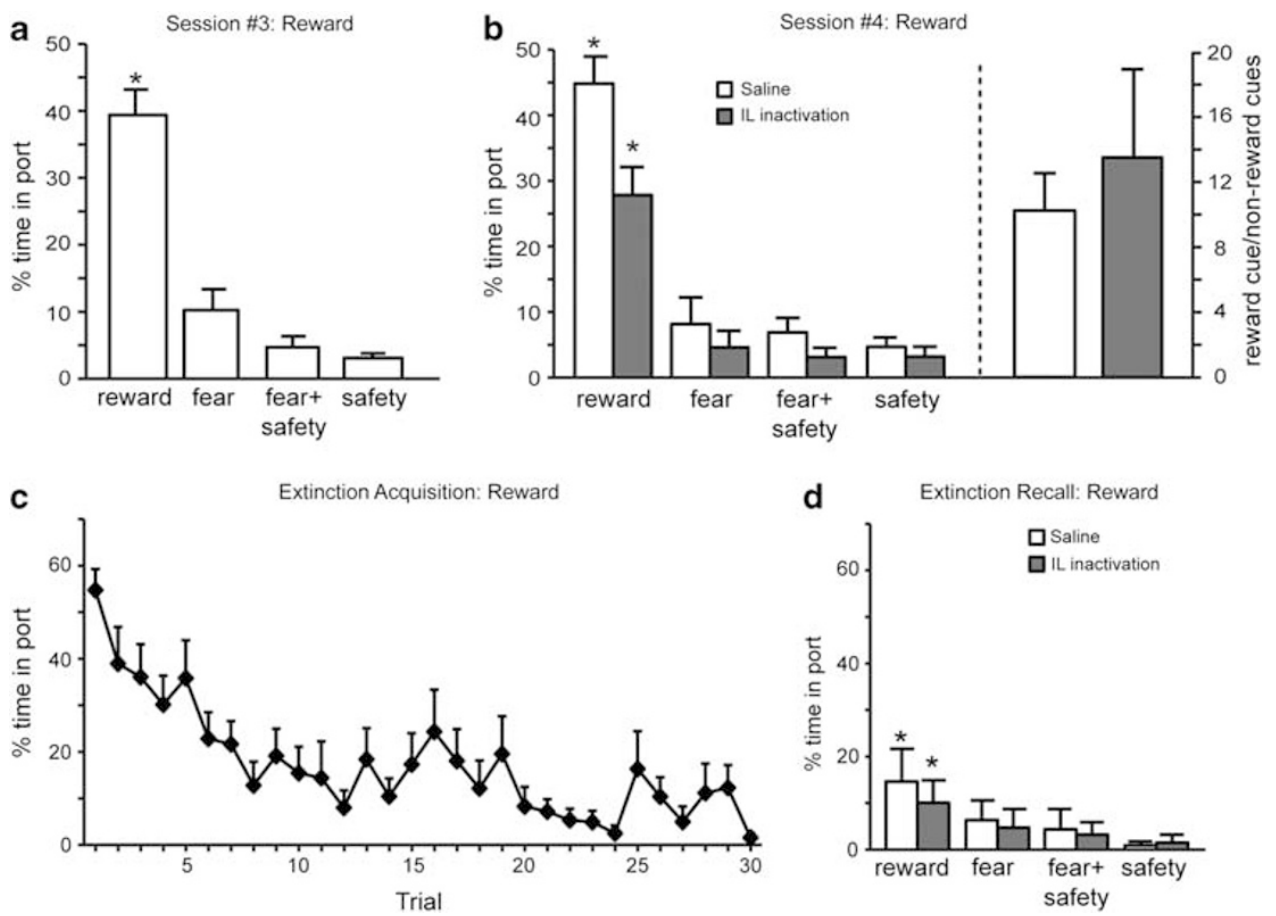

Figure 5 Infralimbic (IL) inactivation does not impair discriminative reward seeking or recall of reward extinction. (a) Discriminative reward seeking under drug-free conditions in session 3. Percentage of time spent in port was significantly higher during the reward cue compared with all other cues (* $p<0.05$ ) under drug-free conditions. (b) (left) Saline-treated rats spent significantly more time in the port during the reward cue compared with all other cues $(* p<0.05)$. IL-inactivated rats also spent significantly more time in port during the reward cue compared with all other cues $(* p<0.05)$. (b) (right) The ratio of time spent in port during the reward cue vs non-reward cues was calculated for saline-treated and IL-inactivated rats in order to assess the degree of discriminative reward seeking. The ratio for saline-treated rats was not significantly different than IL-inactivated rats, indicating similar levels of discrimination. (c) Significant within-session extinction of reward-seeking behavior to the reward cue under drug-free conditions. (d) During extinction recall, both salinetreated and IL-inactivated rats showed a significant reduction in reward seeking to the reward cue compared with the beginning of extinction acquisition $(* p<0.05)$, demonstrating good reward extinction recall.

freezing occurred during the fear + safety cue than when the fear cue as presented alone when rats were infused with saline, but not M/B, into the IL (post-hoc, $p<0.05$ ). Rats demonstrated strong within-session extinction of reward seeking during extinction acquisition (Figure $5 \mathrm{c}$; paired $t$-tests, $p<0.05)$. Following both saline infusions and IL inactivation before extinction recall, reward seeking during the reward cue was significantly reduced compared with the beginning of extinction acquisition (paired $t$-tests, $p<0.05$ ), demonstrating good reward extinction recall. No differences in reward seeking were detected between treatments for any cue during the tests for extinction recall (Figure 5d; main effect of treatment: $\mathrm{F}(1,11)=0.22, p=0.65$; main effect of cue: $\mathrm{F}(3,33)=2.41, p=0.985 ; \mathrm{F}(3,33)=0.20, p=0.90)$.

\section{DISCUSSION}

Using a discriminative Pavlovian conditioning task, we uncovered alterations in reward, fear, and safety cue discrimination after inactivation of the PL and IL cortices: PL contributes to discriminative reward-seeking behavior while IL regulates discrimination between fear and safety cues.

Two previous studies observed unaffected behavioral responses to safety signals after lesions or inactivations of the vmPFC that encompassed both the PL and IL
(Christianson et al, 2008; Gewirtz et al, 1997). Using a different task design than used previously, our results suggest that the rat vmPFC is involved in processing safety signals; however, it is in a sub-region-specific manner. Similar to the methods used by others (Marquis et al, 2007; Willcocks and McNally, 2013), we specifically targeted infusions to the PL and IL sub-regions of the vmPFC. Given the relatively small size of the PL and IL in the rat brain, the potential for spread of the $\mathrm{M} / \mathrm{B}$ to affect both structures is a concern. If the PL infusions involved spread into the IL, or vice versa, we would expect to see the same behavioral effects in both groups of rats. Instead, our study yielded differential roles of these sub-regions in fear/safety behavior. In addition, the novel discriminative task design enabled us to also assess reward-seeking behavior after reversibly inactivating the PL and IL separately. By directly comparing behavioral responses to learned reward, fear, and safety cues, we quantified the ability of rats to seek rewards under appropriate conditions; ie, in the absence of a direct threat and in the presence of reward availability. The task also allows for measurements of the rat's ability to precisely regulate fear in response to cues that predict an aversive event and those that predict the absence of that event.

Conflicting results demonstrating impaired (Laurent and Westbrook, 2009; Sierra-Mercado et al, 2006) and facilitated (Akirav et al, 2006) fear extinction after vmPFC 
inactivations have obscured conclusions regarding the vmPFC's role in fear inhibition. Recent reports that PL mediates fear expression, whereas IL mediates fear reduction (Sierra-Mercado et al, 2011; Vidal-Gonzalez et al, 2006) have shed light on this controversy. Similar to SierraMercado et al (2011), we also observed impaired fear expression during PL inactivation and impaired recall of fear extinction during IL inactivation. In addition, we observed low levels of freezing during PL inactivation to all the cues presented. Most reports utilize a single fear cue and do not compare behavior among different cues. Our study, in contrast, directly compared fear behavior in response to explicit fear and safety cues and found that, in addition to mediating fear reduction to an extinguished fear cue, IL also mediates discrimination between fear and safety cues. During the discriminative conditioning session in which the IL was inactivated (Session \#4, Figure 4b), there was reduced freezing to the fear cue compared with controls, which is in contrast to the high freezing during the fear cue and the compound fear + safety cue during extinction recall (Figure 4d). Additional experiments are necessary to verify the reliability of this effect.

We argue that the PL and IL have different roles in cue discrimination leading to differences in fear and reward behaviors following their inactivation. It is possible that the PL and IL instead have differential roles directly on fear and reward-related behaviors and not on cue discrimination. More specifically, as PL inactivation decreased freezing, it may have enabled increased reward seeking. However, if this were true there should have been equally high rewardseeking behavior during the reward cue when the PL was inactivated compared with saline. We did not observe this; instead, there was decreased reward seeking to the reward cue during PL inactivation. Thus our results demonstrate that PL inactivation affects cue discrimination, an interpretation consistent with the reported role of the PL in modulating attention toward cues during learning (Sharpe and Killcross, 2012) and discrimination among multiple reward cues (Marquis et al, 2007; Mihindou et al, 2013). Our data extends the PL's role to discriminating between dangerous and safe conditions and executing reward seeking behavior under appropriate conditions. As PL inactivation impaired fear expression, it is difficult to determine whether discriminative fear behavior was also affected by the inactivation. PL inactivation significantly reduced the ratio of reward seeking to the reward cue $v s$ non-reward cues as compared with saline treatment, and thus we conclude that discriminative reward seeking was negatively altered by PL inactivation. It is possible that a more global impairment in cue discrimination affecting both discriminative reward seeking behavior and discriminative fear behavior occurs when the PL is inactivated, and future studies should attempt to address this possibility. Our data are also consistent with the report that IL inactivation impairs reward collection (Burgos-Robles et al, 2013). However, in contrast to what was observed during PL inactivation, IL inactivation did not impair discriminative reward seeking as reward seeking increased to the reward cue compared with the other cues during IL inactivation.

The dissociable roles of the PL and IL on fear and reward behaviors are most likely due to their differential connectivity
(Brown and Bowman, 2002; Heidbreder and Groenewegen, 2003; Kesner and Churchwell, 2011; Uylings et al, 2003; Vertes, 2006). PL fibers robustly innervate the nucleus accumbens (Vertes, 2004), an area that regulates goaldirected behaviors (Goto and Grace, 2008). When we inactivated the PL, we saw inappropriate reward-seeking behavior, implying that the impaired discriminative reward seeking could be due to disrupted activity in the PL-nucleus accumbens pathway. PL fibers also project to the central amygdala and the basolateral amygdala, whereas IL fibers project more robustly to the intercalated cells of the amygdala and the lateral portion of the central amygdala (Vertes, 2004). PL projections to the basolateral amygdala may drive fear expression by increasing excitatory drive to the central amygdala; the output of the central amygdala may be inhibited by IL projections to the intercalated cells of the amygdala, thereby reducing fear (Vidal-Gonzalez et al, 2006). Sangha et al (2013) reported separate neuronal populations in the basolateral amygdala selectively responsive to reward, fear, or safety cues. The downstream projections of these neuronal populations are unknown. As specific neurons in the basolateral amygdala respond to safety cues (Sangha et al, 2013) and the IL regulates discrimination between fear and safety cues, as we report here, inputs from the basolateral amygdala and IL may converge upon the intercalated cells of the amygdala and/or lateral portion of the central amygdala to regulate the specificity of the fear response. Both areas of the amygdala have the capability to inhibit the final output of the amygdala, thus promoting fear reduction (Ciocchi et al, 2010; Pape and Pare, 2010; Tye et al, 2011).

The inability to discriminate among cues can lead to generalized fear responses that are enhanced in PTSD patients (Jovanovic et al, 2009), and generalized reward seeking that may contribute to relapse in drug-addicted individuals. We show that inhibiting IL output impaired fear and safety cue discrimination while leaving discriminative reward seeking intact. Inhibiting PL output reduced fear but reduced discriminative reward seeking. Thus methods designed to increase the activity of the human homolog of the rodent IL, and not the PL, may be of therapeutic advantage to PTSD patients. In light of the high comorbidity between PTSD and substance abuse disorders (Kessler et al, 2005), our findings may also be applicable to PTSD patients struggling with drug addictions.

\section{FUNDING AND DISCLOSURE}

This work was supported by a Discovery Grant from the Natural Sciences and Engineering Research Council of Canada (NSERC) and Operating Grant from the Canadian Institutes for Health Research to JGH. PDR was supported by an NSERC Undergraduate Student Research Award. JGH is a Canadian Institutes of Health Research New Investigator. The authors declare no conflict of interest.

\section{ACKNOWLEDGEMENTS}

We thank Brendan G Murray for contributing to behavioral scoring. 


\section{REFERENCES}

Akirav I, Raizel H, Maroun M (2006). Enhancement of conditioned fear extinction by infusion of the GABA(A) agonist muscimol into the rat prefrontal cortex and amygdala. Eur J Neurosci 23: 758-764.

Blanchard RJ, Blanchard DC (1969). Crouching as an index of fear. J Comp Physiol Psychol 67: 370-375.

Brown VJ, Bowman EM (2002). Rodent models of prefrontal cortical function. Trends Neurosci 25: 340-343.

Burgos-Robles A, Bravo-Rivera H, Quirk GJ (2013). Prelimbic and infralimbic neurons signal distinct aspects of appetitive instrumental behavior. PLoS One 8: e57575.

Burgos-Robles A, Vidal-Gonzalez I, Quirk GJ (2009). Sustained conditioned responses in prelimbic prefrontal neurons are correlated with fear expression and extinction failure. J Neurosci 29: 8474-8482.

Cazakoff BN, Howland JG (2011). AMPA receptor endocytosis in rat perirhinal cortex underlies retrieval of object memory. Learn Mem 18: 688-692.

Christianson JP, Benison AM, Jennings J, Sandsmark EK, Amat J, Kaufman RD et al (2008). The sensory insular cortex mediates the stress-buffering effects of safety signals but not behavioral control. J Neurosci 28: 13703-13711.

Christianson JP, Fernando AB, Kazama AM, Jovanovic T, Ostroff LE, Sangha S (2012). Inhibition of fear by learned safety signals: a mini-symposium review. J Neurosci 32: 14118-14124.

Ciocchi S, Herry C, Grenier F, Wolff SB, Letzkus JJ, Vlachos I et al (2010). Encoding of conditioned fear in central amygdala inhibitory circuits. Nature 468: 277-282.

Corcoran KA, Quirk GJ (2007). Activity in prelimbic cortex is necessary for the expression of learned, but not innate, fears. J Neurosci 27: 840-844.

Gewirtz JC, Falls WA, Davis M (1997). Normal conditioned inhibition and extinction of freezing and fear-potentiated startle following electrolytic lesions of medical prefrontal cortex in rats. Behav Neurosci 111: 712-726.

Goto Y, Grace AA (2008). Limbic and cortical information processing in the nucleus accumbens. Trends Neurosci 31: 552-558.

Heidbreder CA, Groenewegen HJ (2003). The medial prefrontal cortex in the rat: evidence for a dorso-ventral distinction based upon functional and anatomical characteristics. Neurosci Biobehav Rev 27: 555-579.

Jovanovic T, Norrholm SD, Fennell JE, Keyes M, Fiallos AM, Myers $\mathrm{KM}$ et al (2009). Posttraumatic stress disorder may be associated with impaired fear inhibition: relation to symptom severity. Psychiatry Res 167: 151-160.

Kesner RP, Churchwell JC (2011). An analysis of rat prefrontal cortex in mediating executive function. Neurobiol Learn Mem 96: 417-431.

Kessler RC, Chiu WT, Demler O, Merikangas KR, Walters EE (2005). Prevalence, severity, and comorbidity of 12-month DSMIV disorders in the National Comorbidity Survey Replication. Arch Gen Psychiatry 62: 617-627.

Killcross S, Coutureau E (2003). Coordination of actions and habits in the medial prefrontal cortex of rats. Cereb Cortex 13: 400-408.

Laurent V, Westbrook RF (2009). Inactivation of the infralimbic but not the prelimbic cortex impairs consolidation and retrieval of fear extinction. Learn Mem 16: 520-529.

Marquis JP, Killcross S, Haddon JE (2007). Inactivation of the prelimbic, but not infralimbic, prefrontal cortex impairs the contextual control of response conflict in rats. Eur J Neurosci 25: $559-566$.

McFarland K, Kalivas PW (2001). The circuitry mediating cocaineinduced reinstatement of drug-seeking behavior. J Neurosci 21: $8655-8663$

Mihindou C, Guillem K, Navailles S, Vouillac C, Ahmed SH (2013). Discriminative inhibitory control of cocaine seeking involves the prelimbic prefrontal cortex. Biol Psychiatry 73: 271-279.

Morrow JD, Maren S, Robinson TE (2011). Individual variation in the propensity to attribute incentive salience to an appetitive cue predicts the propensity to attribute moativational salience to an aversive cue. Behav Brain Res 220: 238-243.

Pape HC, Pare D (2010). Plastic synaptic networks of the amygdala for the acquisition, expression, and extinction of conditioned fear. Physiol Rev 90: 419-463.

Paxinos G, Watson C (1997). The Rat Brain in Stereotaxic Coordinates. Elsevier: Amsterdam, The Netherlands.

Pecina S, Schulkin J, Berridge KC (2006). Nucleus accumbens corticotropin-releasing factor increases cue-triggered motivation for sucrose reward: paradoxical positive incentive effects in stress? BMC Biol 4: 8 .

Rauch SL, Shin LM, Phelps EA (2006). Neurocircuitry models of posttraumatic stress disorder and extinction: human neuroimaging research-past, present, and future. Biol Psychiatry 60: 376-382.

Sangha S, Chadick JZ, Janak PH (2013). Safety encoding in the basal amygdala. J Neurosci 33: 3744-3751.

Sharpe MJ, Killcross S (2012). The prelimbic cortex contributes to the down-regulation of attention toward redundant cues. Cereb Cortex 24: 1066-1074.

Sierra-Mercado D Jr., Corcoran KA, Lebron-Milad K, Quirk GJ (2006). Inactivation of the ventromedial prefrontal cortex reduces expression of conditioned fear and impairs subsequent recall of extinction. Eur J Neurosci 24: 1751-1758.

Sierra-Mercado D, Padilla-Coreano N, Quirk GJ (2011). Dissociable roles of prelimbic and infralimbic cortices, ventral hippocampus, and basolateral amygdala in the expression and extinction of conditioned fear. Neuropsychopharmacology 36: 529-538.

St Onge JR, Floresco SB (2010). Prefrontal cortical contribution to risk-based decision making. Cereb Cortex 20: 1816-1828.

Tye KM, Prakash R, Kim SY, Fenno LE, Grosenick L, Zarabi H et al (2011). Amygdala circuitry mediating reversible and bidirectional control of anxiety. Nature 471: 358-362.

Uylings HB, Groenewegen HJ, Kolb B (2003). Do rats have a prefrontal cortex? Behav Brain Res 146: 3-17.

Van den Oever MC, Spijker S, Smit AB, De Vries TJ (2010). Prefrontal cortex plasticity mechanisms in drug seeking and relapse. Neurosci Biobehav Rev 35: 276-284.

Vertes RP (2004). Differential projections of the infralimbic and prelimbic cortex in the rat. Synapse 51: 32-58.

Vertes RP (2006). Interactions among the medial prefrontal cortex, hippocampus and midline thalamus in emotional and cognitive processing in the rat. Neuroscience 142: 1-20.

Vidal-Gonzalez I, Vidal-Gonzalez B, Rauch SL, Quirk GJ (2006). Microstimulation reveals opposing influences of prelimbic and infralimbic cortex on the expression of conditioned fear. Learn Mem 13: 728-733.

Willcocks AL, McNally GP (2013). The role of medial prefrontal cortex in extinction and reinstatement of alcohol-seeking in rats. Eur J Neurosci 37: 259-268. 